\title{
Apoio social e prática de atividade física no lazer em adolescentes: um estudo de base populacional
}

Social support and physical activity during leisure in adolescents: a population-based study

\section{AUTORES \\ Márcio Botelho Peixoto ${ }^{1}$ (D) \\ Alan Goularte Knuth ${ }^{1,2}$ (D) \\ Inacio Crochemore Mohnsam da Silva ${ }^{1}$ \\ Pedro Curi Hallal ${ }^{1}$ (D) \\ 1 Universidade Federal de Pelotas, Programa de Pós-Graduação em Epidemiologia, Departamento de Medicina Social, Pelotas, Rio Grande do Sul, Brasil. \\ 2 Universidade Federal do Rio Grande, Instituto de Educação, Programa de Pós-Graduação em Saúde Pública, Residência Multiprofissional em Saúde da Família, Rio Grande, Rio Grande do Sul, Brasil.}

\section{CONTATO}

Márcio Botelho Peixoto

marcio_b_peixoto@hotmail.com

Rua Hebert de Sousa, n. 918, Pelotas, Rio

Grande do Sul, Brasil. CEP: 96085-282.

DOI

$10.12820 /$ rbafs. $24 \mathrm{e} 0103$

\section{(cc) BY-NC-SA}

Este obra está licenciado com uma Licença Creative Commons Atribuicão-NãoComercialCompartilhalgual 4.0 Internacional.

\begin{abstract}
RESUMO
O objetivo do estudo foi avaliar a associação entre a prática de atividade física (AF) no lazer e o apoio social em adolescentes de 10 a 19 anos. O estudo transversal, de base populacional, foi realizado em Pelotas, Rio Grande do Sul, Brasil. Foram considerados ativos os adolescentes que realizavam no mínimo 300 minutos semanais de $\mathrm{AF}$ no lazer. $\mathrm{O}$ apoio social foi estudado através da Escala de Apoio Social para a AF. Foram entrevistados 743 adolescentes. A proporção de ativos no lazer foi de 28,2\% (IC 95\%: 24,6-31,3). Meninos com maior apoio social de amigos apresentaram uma probabilidade 112\% (IC 95\%: 41,0-183,0) maior de serem ativos em comparação a seus pares com menor apoio social. Meninas com apoio social elevado de familiares tiveram uma probabilidade de 102\% (IC 95\%: 12,0-193,0) maior de serem ativas. O percentual equivalente entre os meninos foi de 44\% (IC 95\%: 8,0-103,0). O incentivo de familiares e amigos exerce influência na prática de AF e apresenta questões culturais, como a indução de meninas para caminhadas e meninos para outras AF, especialmente dos amigos.
\end{abstract}

Palavras-chave: Atividade motora; Adolescentes; Apoio social; Estudo transversal.

\section{ABSTRACT}

The aim of the study was to evaluate the association between leisure-time physical activity (PA) and social support among adolescents aged 10 to 19 years. This was a population-based cross-sectional study carried out in the city of Pelotas, Rio Grande do Sul, Brazil. Adolescents were considered as active in leisure-time if reporting to practice $300 \mathrm{~min} /$ wh or more of PA. Social support was assessed through the PA Social Support Scale. The study included 743 adolescents. The proportion of active adolescents was 28.2\% (95\%CI: 24.631.3). Boys who report to have higher social support from friends were 112\% (95\%CI: 41.0-214.0) more likely to achieve PA recommendations as compared to their peers reporting no such social support. Girls with high scores of social support from the family were 102\% (95\% CI: 12.0-193.0) more likely to be active than those without such a support. The equivalent proportion for boys was 44\% (95\%CI: 8.0-103.0). The encouragement of family and friends influences the practice of $P A$ and presents cultural issues, such as inducing girls to walk and boys to other $P A$ s, especially friends.

Keywords: Motor activity; Adolescents; Social support; Cross-sectional study.

\section{Introdução}

Cerca de quatro quintos da população de 13 a 15 anos no mundo não alcançam a recomendação da Organização Mundial da Saúde referente à prática de atividade física $^{1,2}$. A cadeia de determinação da prática de atividade física é complexa, envolvendo aspectos políticos, ambientais, culturais, sociais e demográficos ${ }^{3}$. Existe uma concentração de estudos avaliando a associação entre fatores sociodemográficos e a prática de atividade física ${ }^{4}$, mas ainda são explorados em menor quantidade os dados sobre a influência dos demais aspectos que influenciam esse comportamento, especialmente em países de rendas média e baixa ${ }^{3}$.

Entre os determinantes sociais, o apoio social, que compreende os recursos fornecidos por outras pessoas para a completa execução de algum comportamento ${ }^{5}$, apresenta-se como um aspecto relevante no estudo da atividade física. Dois estudos de revisão publicados encontraram associação entre apoio social e prática de atividade física em adolescentes; aqueles que recebiam apoio social apresentaram maior probabilidade de serem ativos fisicamente ${ }^{6,7}$.

Cabe salientar que existem distintas compreensões sobre apoio social e que este conceito sofre variações ou adaptações conforme o contexto em que é estudado. Por exemplo, entre os diferentes domínios de apoio social, Vrazel et a ${ }^{8}$ categorizam-no em emocional, tangível e informacional. $\mathrm{O}$ apoio social emocional aborda 
o quanto os indivíduos são acompanhados ou encorajados a mudar seu comportamento pelas pessoas que integram o seu convívio social. As questões financeiras e logísticas estão colocadas no domínio tangível, sendo caracterizado pela ajuda prática para a realização de atividades físicas, como transporte e custeio. O domínio informacional é definido como o recebimento de informação sobre os benefícios de uma determinada modificação de comportamento, por meio de indivíduos de seu convívio social. Além dos domínios, também há a caracterização do apoio social com respeito às fontes, que podem ser a família ou os amigos. Alguns estudos indicam que a relação de proximidade de quem fornece o apoio social potencializa a mudança de comportamento do indivíduo que o recebe ${ }^{9-10}$.

Neste contexto, identificar aspectos que potencialmente influenciam a prática de atividade física em adolescentes, especialmente em países de renda baixa e média, são importantes para embasar futuras estratégias de promoção desse comportamento. Assim, o presente estudo tem como objetivo descrever a associação entre apoio social de familiares e amigos e a prática de atividade física no lazer em adolescentes.

\section{Métodos}

Este estudo fez parte uma pesquisa sobre saúde idealizada pelo Programa de Pós-Graduação em Epidemiologia da Universidade Federal de Pelotas (UFPel). Utilizou-se um delineamento transversal, de base populacional, na zona urbana de Pelotas, no sul do Rio Grande do Sul, durante o primeiro semestre de 2012. Embora o presente artigo seja específico à população adolescente, a amostra geral foi composta também por adultos e idosos, os quais responderam a um questionário constituído de informações demográficas, socioeconômicas, comportamentais e de saúde.

O processo de amostragem listou os 495 setores censitários da cidade e os ordenou pela sua numeração, de acordo com os dados do Censo de $2010^{11}$. Essa estratégia é baseada na localização geográfica dos setores, numerados em uma ordem em formato espiral, do centro para as periferias. Tal medida foi adotada para garantir a participação de diversos bairros da cidade e, assim, de diferentes contextos socioeconômicos. A partir de um número aleatório sorteado no programa Stata/IC 12.0, foram selecionados, sistematicamente, 130 setores, respeitando a probabilidade proporcional ao número de domicílios dos mesmos. O número de domicílios selecionados em cada setor variou de 11 a 36, totalizando 1.723 domicílios.
Para atender aos objetivos do presente estudo, foram entrevistados os adolescentes com idade entre 10 e 19 anos $^{12}$. Os critérios de exclusão adotados foram incapacidade mental para responder o questionário, institucionalização (por exemplo, Fundação de Atendimento Socioeducativo e presídio municipal) ou incapacidade física severa que impedia de forma permanente a prática de atividade física ou nos sete dias anteriores a entrevista. Foram considerados como perdas ou recusas os adolescentes que não foram localizados após três visitas ao domicílio ou aqueles que não concordaram em participar do estudo, respectivamente.

Foram realizados cálculos de tamanho de amostra para o estudo da prevalência de atividade física no lazer, prevalência de apoio social em cada domínio e associação entre atividade física e apoio social. O cálculo que requeria o maior tamanho amostral utilizou os seguintes parâmetros e estimativas: nível de confiança de 95\% e margem de erro tolerada de cinco pontos percentuais. Em relação ao apoio social foi considerado a prevalência de $33 \%$ do artigo publicado em adultos da mesma cidade da coleta ${ }^{13}$. Após, foi adicionado $10 \%$ para perdas e recusas, e considerando um efeito de delineamento de 1,5, eram necessários 678 indivíduos. O cálculo do poder estatístico desta amostra foi feito posteriormente, considerando prevalência da atividade física entre os não expostos de $45 \%$, intervalo de confiança de $95 \%$ e poder de $80 \%$, foi possível detectar razões de prevalência iguais ou superiores a 1,36 e iguais ou inferiores a 0,84 .

Para a coleta do nível de atividade física foi aplicado um questionário para adolescentes, o qual teve sua reprodutibilidade testada previamente por Bastos et $\mathrm{al}^{14}$. O instrumento apresenta uma boa correlação entre teste e reteste (rho 0,62), evidenciando que $73 \%$ dos indivíduos obtiveram a mesma classificação do questionário. Este instrumento aborda a atividade física de lazer por meio de uma lista de atividades para as quais, quando realizadas na última semana, eram registradas a frequência semanal e o tempo médio gasto em cada uma delas. Cabe ressaltar que o referido artigo traz evidências de validade do questionário quando comparado com pedômetro (rho de Spearman =0,26; coeficiente Kappa de concordância de 0,18 ; sensibilidade $=77,1 \%$ e especificidade $=42,6 \%)^{14}$.

Adicionalmente, realizaram-se análises avaliando a prática da caminhada no período de lazer como desfecho e o apoio social para essa prática, independentemente da frequência e da duração. 
Os entrevistados foram incluídos em uma das três categorias: adolescentes que não realizaram atividade física de lazer na última semana ("zero minuto"); adolescentes que realizaram de "1-299 minutos" de atividade física de lazer; e os que realizaram "300 minutos ou mais" de atividade física de lazer nos últimos sete dias. Foram considerados fisicamente ativos os indivíduos que realizavam 300 minutos ou mais por semana de atividades físicas no lazer². Não foram avaliadas as atividades físicas de deslocamento e aquelas realizadas durante as aulas de educação física.

O apoio social foi estudado através da Escala de Apoio Social para a Atividade Física $(\mathrm{EASAF})^{15}$, a qual é composta por seis questões, que foram duplicadas de maneira a formarem dois blocos, sendo o primeiro bloco relacionado ao apoio social para caminhada e o segundo para o apoio social relacionado às demais atividades físicas no lazer. A validade da EASAF foi avaliada através da Correlação Ordenada de Spearmann e os valores de alfa variaram de 0,78 a 0,81 e os coeficientes Intraclasse de 0,61 a 0,81, evidenciando a fidedignidade adequada e validade moderada para o uso em adolescentes Brasileiros ${ }^{15}$. Em cada bloco, os respondentes relataram a frequência do recebimento de apoio social de familiares ou de amigos, desde: "fizeram junto", "convidaram" ou "incentivaram" a prática de caminhada e atividades físicas no lazer, nos últimos três meses. Cada pergunta apresenta três opções de resposta: "(0) nunca”, "(1) às vezes" e "(2) sempre”. Os escores de apoio social foram determinados pela soma das respostas de cada bloco, que varia de zero a seis pontos. Para a análise de cada bloco de apoio social (apoio social para caminhada e para outras atividades físicas de lazer) foi construída uma variável somando as três questões do bloco e com uma variação de zero a 24 pontos, de cada uma das possíveis fontes (familiares ou amigos). Complementarmente, essa variável foi analisada em tercis, na qual o terceiro tercil foi considerado como grupo exposto.

As variáveis exploradas para fins de ajustes foram faixa etária (10-13, 14-16 e 17-19 anos) e classe econômica $(\mathrm{A} / \mathrm{B}, \mathrm{C}, \mathrm{D} / \mathrm{E})$ baseada em índices de bens e escolaridade do chefe da família, de acordo com a classificação da Associação Brasileira de Empresas de Pesquisas $(\mathrm{ABEP})^{16}$. Todas as análises foram estratificadas por sexo e o teste de qui-quadrado para heterogeneidade foi utilizado para detectar diferenças estatisticamente significativas entre meninos e meninas na descrição da amostra e na frequência do recebimento de apoio social. Para a associação entre o apoio social e a atividade física foram desenvolvidos modelos de regressão de Poisson, com ajuste robusto da variância. $\mathrm{O}$ nível de significância adotado foi de $5 \%$ e, em todas as análises, o efeito de delineamento foi considerado. Todas as análises foram realizadas no programa estatístico STATA/IC 12.0.

O estudo foi aprovado pelo Comitê de Ética em Pesquisa da Faculdade de Medicina da UFPel e protocolado sob o número OF. 77/11. Todos os participantes assinaram o Termo de Consentimento Livre e Esclarecido.

\section{Resultados}

Foram visitados 1.723 domicílios, nos quais foram identificados 786 adolescentes para o estudo. Do total de elegíveis, 43 se recusaram a participar da pesquisa (5,5\%; 30 meninos e 13 meninas). Da amostra estudada, $50,5 \%$ eram meninas e $75,5 \%$ dos adolescentes se caracterizaram como de cor de pele branca. A idade média da amostra foi de 14,6 anos (desvio padrão = 0,11), e $11,1 \%$ dos entrevistados foram classificados como de classe econômica D - E (Tabela 1).

Tabela 1 - Descrição da amostra conforme características demográficas, socioeconômicas. Pelotas, Rio Grande do Sul, 2012 ( $\mathrm{n}=743$ )

\begin{tabular}{lccc}
\hline Variáveis em estudo & Total & Meninos & Meninas \\
\cline { 2 - 4 } & $\mathrm{n}(\%)$ & $\mathrm{n}(\%)$ & $\mathrm{n}(\%)$ \\
\hline Características amostrais & & & \\
Adolescentes identificados & $786(100)$ & $389(49,5)$ & $397(50,5)$ \\
Adolescentes entrevistados & $743(94,5)$ & $359(92,3)$ & $384(96,7)$ \\
Perdas e recusas & $43(5,5)$ & $30(7,7)$ & $13(3,3)$ \\
Cor da pele & & & \\
Branca & $561(75,5)$ & $275(75,8)$ & $289(75,3)$ \\
Preta & $109(14,7)$ & $58(16,2)$ & $51(13,4)$ \\
Parda & $65(8,7)$ & $23(6,4)$ & $42(10,9)$ \\
Amarela & $6(0,8)$ & $5(1,4)$ & $1(0,2)$ \\
Indígena & $2(0,3)$ & $1(0,2)$ & $1(0,2)$ \\
Idade & & & \\
10 -13 & $280(37,7)$ & $146(40,7)$ & $134(34,9)$ \\
$14-16$ & $217(29,2)$ & $106(29,5)$ & $111(28,9)$ \\
$17-19$ & $246(33,1)$ & $107(29,8)$ & $139(36,2)$ \\
Classe econômica & & & \\
A - B & $31(11,1)$ & $40(11,4)$ & $41(10,8)$ \\
C & $307(41,9)$ & $136(38,6)$ & $171(45,0)$ \\
D - E & $344(47,0)$ & $176(50,0)$ & $168(44,2)$ \\
\hline & & &
\end{tabular}

A Figura 1 apresenta a prevalência de atividade fí- 
sica de lazer na amostra total e de acordo com o sexo dos entrevistados. A proporção de ativos no lazer foi de 28,2\% (IC95\%: 24,6-31,3). A proporção de ativos foi $66 \%$ menor entre as meninas quando comparada com os meninos. A distribuição de atividade física na categoria intermediária (1-299 minutos por semana) foi bastante similar entre os sexos. A proporção de jovens que relatou zero minuto por semana de prática de atividade física de lazer foi 55,6\% superior nas meninas em comparação aos meninos.

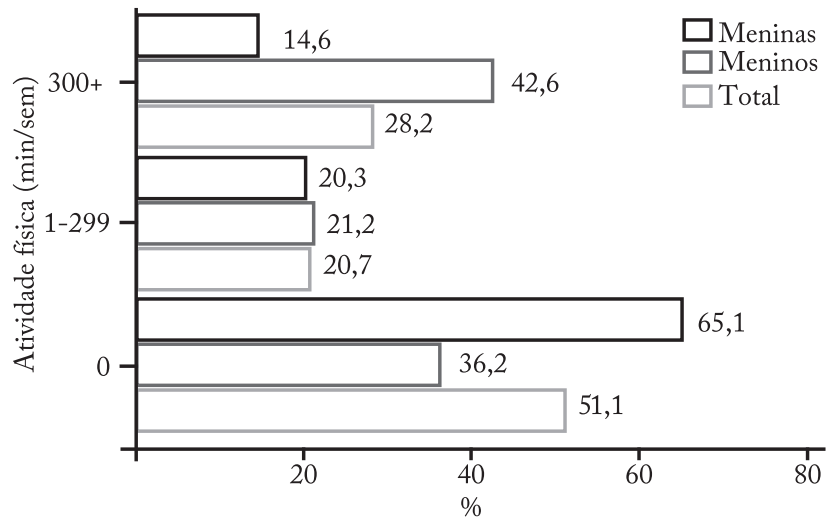

Figura 1 - Níveis de atividade física em adolescentes. Pelotas, Rio Grande do Sul, 2012 ( $\mathrm{n}=743$ ).
A Tabela 2 descreve a frequência de apoio social entre os adolescentes. $\mathrm{O}$ apoio social para caminhadas foi sempre maior entre as meninas, comparadas aos meninos, tanto no bloco familiar quanto no que se refere ao apoio de amigos. Entretanto o apoio social de amigos para a prática de outras atividades físicas foi maior nos meninos do que nas meninas, sendo que eles recebem mais convites de seus amigos para praticar atividades físicas. Entre os familiares, não houve diferença estatisticamente significativa no apoio social para outras atividades entre meninos e meninas.

A Figura 2 apresenta a associação entre prática de caminhada ( $\operatorname{sim} /$ não) e apoio social de amigos e familiares específicos para esta prática, enquanto a Figura 3 descreve a associação entre o apoio social para outras atividades físicas no lazer e a prática de atividade física no lazer. Dentre as diferentes fontes de apoio social, o apoio de amigos para caminhadas esteve associado com a prática de caminhada, tanto em meninas quanto em meninos. Contudo o apoio social de familiares esteve associado apenas entre as meninas (Figura 2). Por outro lado, o apoio social para a prática de outras atividades físicas mostrou-se significativamente associado aos níveis de atividade física da amostra, independente-

Tabela 2 - Descrição da frequência de apoio social em adolescentes nos diferentes domínios de acordo com sexo. Pelotas, Rio Grande do Sul, $2012(n=743)$.

\begin{tabular}{|c|c|c|c|c|c|c|c|}
\hline \multirow[b]{2}{*}{ Apoio social } & \multicolumn{3}{|c|}{ Meninos } & \multicolumn{3}{|c|}{ Meninas } & \multirow[b]{2}{*}{$\mathrm{p}^{*}$} \\
\hline & $\begin{array}{l}\text { Nunca } \\
\mathrm{n}(\%)\end{array}$ & $\begin{array}{c}\text { Às vezes } \\
\mathrm{n}(\%)\end{array}$ & $\begin{array}{c}\text { Sempre } \\
\mathrm{n}(\%)\end{array}$ & $\begin{array}{l}\text { Nunca } \\
\mathrm{n}(\%)\end{array}$ & $\begin{array}{c}\text { Às vezes } \\
\mathrm{n}(\%)\end{array}$ & $\begin{array}{c}\text { Sempre } \\
\mathrm{n}(\%)\end{array}$ & \\
\hline \multicolumn{8}{|l|}{ Caminhada no lazer } \\
\hline \multicolumn{8}{|l|}{ Familiares } \\
\hline Fez caminhada com você & $255(71,0)$ & $90(25,1)$ & $14(3,9)$ & $196(51,0)$ & $163(42,5)$ & $25(6,5)$ & $<0,001$ \\
\hline Convidou você para caminhar & $220(61,3)$ & $121(33,7)$ & $18(5,0)$ & $72(44,8)$ & $179(46,6)$ & $33(8,6)$ & $<0,001$ \\
\hline Incentivou você a caminhar & $212(59,1)$ & $106(29,5)$ & $41(11,4)$ & $158(41,1)$ & $132(34,4)$ & $94(24,5)$ & $<0,001$ \\
\hline \multicolumn{8}{|l|}{ Amigos } \\
\hline Fez caminhada com você & $216(60,2)$ & $102(28,4)$ & $41(11,4)$ & $212(55,2)$ & $139(36,2)$ & $33(8,6)$ & 0,056 \\
\hline Convidou você para caminhar & $209(58,2)$ & $117(32,6)$ & $33(9,2)$ & $189(49,2)$ & $161(41,9)$ & $34(8,9)$ & 0,028 \\
\hline Incentivou você a caminhar & $267(74,3)$ & $68(19,0)$ & $24(6,7)$ & $227(59,1)$ & $125(32,6)$ & $32(8,3)$ & $<0,001$ \\
\hline \multicolumn{8}{|l|}{ Atividade física no lazer } \\
\hline \multicolumn{8}{|l|}{ Familiares } \\
\hline Fez atividade física com você & $215(59,9)$ & $119(33,2)$ & $25(6,9)$ & $245(63,8)$ & $123(32,0)$ & $16(4,2)$ & 0,206 \\
\hline Convidou você para fazer atividade física & $218(60,7)$ & $116(32,3)$ & $25(7,0)$ & $214(55,7)$ & $144(37,5)$ & $26(6,8)$ & 0,327 \\
\hline Incentivou você a fazer atividade física & $165(45,9)$ & $115(32,0)$ & $79(22,1)$ & $177(46,1)$ & $124(32,3)$ & $83(21,6)$ & 0,991 \\
\hline \multicolumn{8}{|l|}{ Amigos } \\
\hline Fez atividade física com você & $84(23,4)$ & $149(41,5)$ & $126(35,1)$ & $189(49,2)$ & $145(37,8)$ & $50(13,0)$ & $<0,001$ \\
\hline Convidou você para fazer atividade física & $97(27,0)$ & $144(40,1)$ & $118(32,9)$ & $166(43,2)$ & $168(43,8)$ & $50(13,0)$ & $<0,001$ \\
\hline Incentivou você a fazer atividade física & $157(43,7)$ & $112(31,2)$ & $90(25,1)$ & $197(51,3)$ & $139(36,2)$ & $48(12,5)$ & $<0,001$ \\
\hline
\end{tabular}

*Teste de Qui-quadrado para heterogeneidade. 
mente da fonte e do sexo do adolescente, evidenciando que quanto maior o apoio recebido, maior a prevalência de atividade física de lazer (Figura 3).

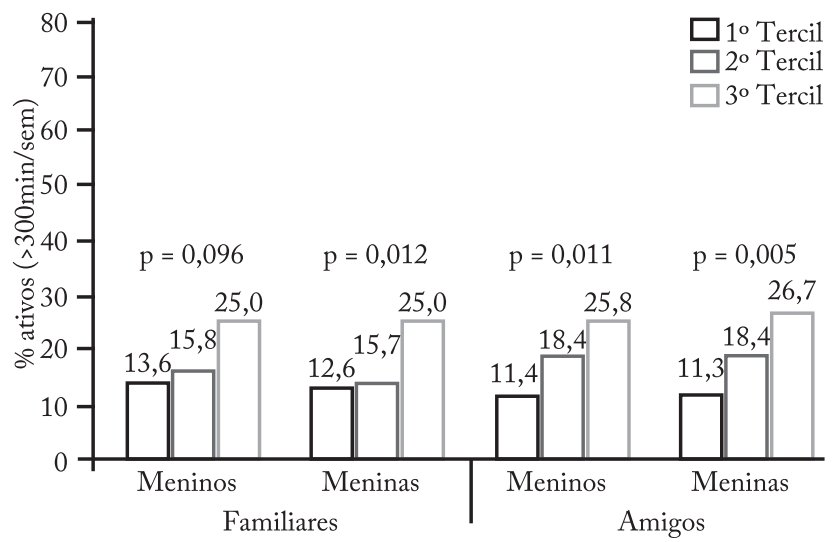

Figura 2 - Associação entre prática de caminhada e apoio social para caminhada de familiares e amigos. Pelotas, Rio Grande do Sul, $2012(\mathrm{n}=743)$

* Teste de Qui-quadrado para heterogeneidade.

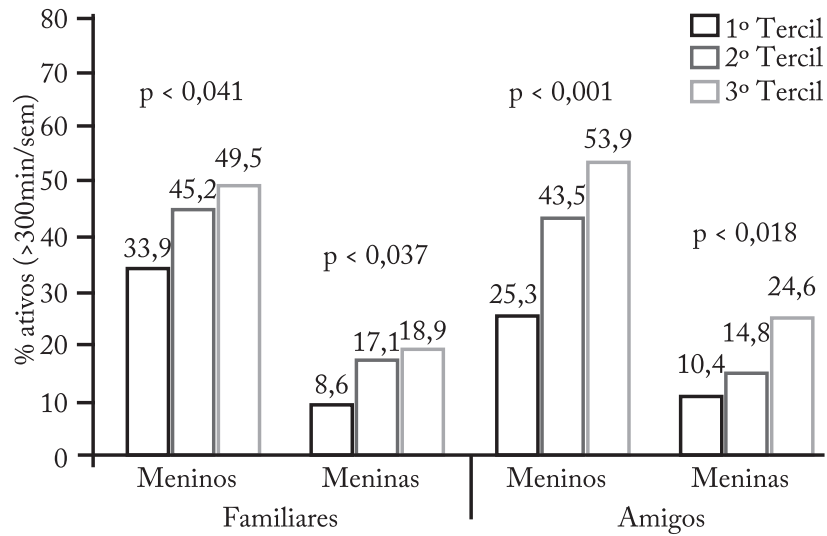

Figura 3 - Associação entre atividade física no lazer $(\geq 300 \mathrm{~min} /$ semana) e apoio social para atividades físicas de familiares e amigos. Pelotas, Rio Grande do Sul, 2012 ( $\mathrm{n}=743$ ).

*Teste de Qui-quadrado para heterogeneidade.
As associações brutas apresentadas nas Figuras 2 e 3 foram reproduzidas em modelos de regressão de Poisson com ajuste para potenciais fatores de confusão ( $\mathrm{Ta}-$ bela 3). Os resultados indicam que meninos e meninas pertencentes ao maior tercil de recebimento de apoio social para caminhadas de familiares têm uma probabilidade cerca de $90 \%$ maior de praticar caminhada no lazer, quando comparados aos grupos referência. Quando avaliado o apoio social oferecido por amigos, o efeito evidenciado foi ainda maior. Os meninos e as meninas pertencentes ao maior tercil de recebimento de apoio social de seus amigos apresentaram uma probabilidade cerca de duas vezes maior de praticarem caminhadas quando estimulados por seus pares (Tabela 3 ).

Com relação a outras atividades físicas de lazer, meninos e meninas com maior apoio social de amigos para a prática de atividade física no lazer (pertencentes ao $3^{\circ}$ tercil) apresentaram uma probabilidade duas vezes maior de realizarem pelo menos 300 minutos semanais de atividade física em comparação a outros adolescentes sem ou com menor apoio social ( $1^{\circ}$ tercil). Considerando o apoio de familiares, entre os meninos, aqueles que com maior apoio apresentaram uma probabilidade $44 \%$ maior de atingir o critério de pelo menos 300 minutos de atividade física por semana em comparação aos meninos que relataram pouco ou nenhum apoio social. Já entre as meninas com maior apoio social de familiares essa probabilidade foi $102 \%$ maior (Tabela 3).

\section{Discussão}

O presente estudo evidenciou uma associação direta do apoio social com a prática de caminhada no lazer e

Tabela 3 - Análise ajustada da associação entre atividade física no lazer e apoio social de amigos e familiares para atividade física e caminhada. Pelotas, Rio Grande do Sul, $2012(\mathrm{n}=743)$

\begin{tabular}{|c|c|c|c|c|c|c|c|c|c|c|c|c|c|c|}
\hline \multirow{4}{*}{ Variáveis } & \multicolumn{4}{|c|}{ Meninos } & \multicolumn{3}{|c|}{ Meninas } & \multicolumn{4}{|c|}{ Meninos } & \multicolumn{3}{|c|}{ Meninas } \\
\hline & $>300$ & \multicolumn{3}{|c|}{ Análise Ajustada* } & \multicolumn{3}{|c|}{ Análise Ajustada* } & $>300$ & \multicolumn{3}{|c|}{ Análise Ajustada* } & \multicolumn{3}{|c|}{ Análise Ajustada* } \\
\hline & $\mathrm{n}(\%)$ & $\mathrm{RP}$ & IC 95\% & $\mathrm{p}$ & $\mathrm{RP}$ & IC 95\% & $\mathrm{p}$ & $\mathrm{n}(\%)$ & $\mathrm{RP}$ & IC 95\% & $\mathrm{p}$ & $\mathrm{RP}$ & IC 95\% & $\mathrm{p}$ \\
\hline & \multicolumn{7}{|c|}{ Apoio social para caminhada no lazer } & \multicolumn{7}{|c|}{ Apoio social para atividade física no lazer } \\
\hline Familiares & & & & 0,026 & & & 0,009 & & & & 0,011 & & & 0,013 \\
\hline $1^{\circ}$ Tercil & $21(13,6)$ & 1,00 & - & & 1,00 & - & & $42(33,9)$ & 1,00 & - & & 1,00 & - & \\
\hline $2^{\circ}$ Tercil & $21(15,8)$ & 1,16 & $0,70-1,93$ & & 1,09 & $0,56-2,13$ & & $57(45,2)$ & 1,35 & $0,96-1,40$ & & 1,91 & $0,99-3,70$ & \\
\hline 3o Tercil & $18(25,0)$ & 1,95 & $1,14-3,36$ & & 1,92 & $1,12-3,28$ & & $59(49,2)$ & 1,44 & $1,08-2,03$ & & 2,02 & $1,12-2,93$ & \\
\hline Amigos & & & & 0,010 & & & 0,004 & & & & $<0,001$ & & & $<0,001$ \\
\hline $1^{\circ}$ Tercil & $20(11,4)$ & 1,00 & & & 1,00 & - & & $23(25,3)$ & 1,00 & - & & 1,00 & - & \\
\hline $2^{\circ}$ Tercil & $18(18,4)$ & 1,56 & $0,85-2,87$ & & 1,55 & $0,83-2,91$ & & $60(43,5)$ & 1,74 & $1,16-2,54$ & & 1,27 & $0,70-2,32$ & \\
\hline $3^{\circ}$ Tercil & $22(25,8)$ & 2,14 & $1,19-4,02$ & & 2,33 & $1,31-4,13$ & & $70(53,9)$ & 2,12 & $1,41-2,83$ & & 2,10 & $1,12-3,93$ & \\
\hline
\end{tabular}

*Regressão de Poisson com ajuste para nível sócio econômico e idade 
com a prática de pelo menos 300 minutos de atividade física semanal no lazer. Os adolescentes pertencentes ao maior tercil de apoio social foram duas vezes mais ativos do que aqueles que relataram receber pouco ou nenhum apoio para atividade física. Essa associação foi observada nos meninos e nas meninas, tanto em relação ao apoio dos amigos quanto ao apoio dos familiares. Em virtude dessas evidências, é relevante também o fato de que cerca de $50 \%$ dos entrevistados não relataram o recebimento de qualquer tipo de apoio social para a prática de atividade física.

Cabe ressaltar a necessidade de reflexão sobre a compreensão da forma em que se configura o apoio social. O esforço do campo epidemiológico em estudar esse aspecto configura uma ampliação nos fatores importantes para a determinação de comportamentos no campo da saúde. No entanto, certamente outros aspectos de difícil mensuração impactam no apoio social e na sua real influência sobre os padrões de atividade física. Ressaltando a objetividade intrínseca ao método, destaca-se que outros tipos de instrumentos ou metodologias precisam ser utilizados para complementar a abordagem epidemiológica no estudo dessa associação.

Nesse sentido, estudos qualitativos poderão auxiliar na melhor compreensão entre a relação do apoio social e a prática de atividade física ${ }^{17,18}$. Assim, a compreensão sobre o apoio social seria ampliada, podendo relevar a intensidade e a especificidade de aspectos culturais na viabilização dessa associação.

No Brasil, investigações anteriores verificaram o papel do apoio social sobre a prática de atividade física ${ }^{19-21}$. O estudo de Reis ${ }^{19}$, utilizando a mesma escala de apoio social com adolescentes de 14 a 17 anos, apontou uma associação positiva com a prática de atividade física em seus diferentes domínios. $\mathrm{O}$ mesmo autor ${ }^{20}$, em outra publicação, detectou associação entre apoio social especificamente de familiares e prática de atividade física apenas nos meninos, corroborando em parte os achados do presente estudo, no qual as associações foram mais consistentes nesse grupo. Um estudo realizado por Ricardo et $\mathrm{al}^{21}$ avaliou uma amostra não representativa de estudantes concluiu que os adolescentes que tiveram um maior apoio social dos pais e também dos amigos tiveram maior probabilidade de serem ativos quando comparados àqueles com menor apoio social.

Figueira $\mathrm{Jr}^{22}$, em um estudo realizado em duas cidades no interior de São Paulo, com adolescentes de 14 a 18 anos, encontraram associação entre prática de atividade física e apoio social de amigos. Outros es- tudos mais recentes também encontraram associação entre a prática de atividade física e o apoio social no sul do Brasil $^{22,23}$. As demais publicações brasileiras sobre o tema têm como enfoque principal a população adulta e são consistentes ao demonstrar associações positivas entre apoio social e atividade física no lazer ${ }^{15,22,24}$.

Os resultados deste trabalho, assim como outras evidências já publicadas ${ }^{20-22}$, demonstram que especificidades da influência do apoio social para a prática de atividades físicas podem ser atribuídas às diferenças de gênero, como por exemplo, o maior efeito do apoio familiar para prática de caminhada entre as meninas em comparação com os meninos. $\mathrm{O}$ fato é que os meninos são mais ativos ao longo da adolescência até a vida adulta ${ }^{1,25}$, e os determinantes deste comportamento podem ser diferentes de acordo com o sexo, destacando a necessidade de avaliação específicas como as apresentadas neste estudo.

Dessa forma, torna-se relevante o entendimento do efeito específico do apoio social de familiares e de amigos entre os adolescentes. Durante a infância, a família parece ser o primeiro e o mais poderoso agente socializador de valores, comportamentos e normas ${ }^{26}$. Talvez por esse motivo um maior efeito do apoio social exista entre as meninas quando o apoio social de familiares é elevado, pois a presença dos pais, o incentivo e convites, podem auxiliar e estimular as meninas a praticarem atividade física no lazer.

Neste estudo, os meninos receberam mais convites de seus amigos, em comparação às meninas, porém a influência dos amigos foi tão positiva entre meninos quanto entre meninas. A influência dos amigos pode ser exercida, por exemplo, de três formas distintas: os adolescentes influenciam-se mutuamente no início de uma atividade; um adolescente pode iniciar uma atividade pelo fato do seu amigo já praticá-la; e relações de amizade são estabelecidas entre adolescentes que estão envolvidos em atividades físicas e desportivas ${ }^{27}$.

Uma limitação do instrumento empregado neste estudo é a avaliação restrita ao apoio social de amigos e familiares em virtude da existência de outras fontes de apoios que não foram medidas, incluindo as influências da mídia, da formação escolar, do contexto cultural e, principalmente, da interação desses aspectos com aqueles medidos pelo presente estudo. Essas interações podem não estar expressas nas respostas dos adolescentes a um questionário objetivo num inquérito epidemiológico. Assim, as interpretações deste estudo são específicas sobre o tipo de apoio social estudado, 
ainda que as interações acima destacadas certamente impactem nas decisões e escolhas referentes a atividades físicas e em outros comportamentos.

Outra limitação que merece reflexão é a falta de uma avaliação de apoio social tangível, como o pagamento de aulas e espaços para a prática de atividade física e aspectos logísticos como a condução dos jovens para locais de prática. Esses apoios podem ser especificamente importantes na faixa etária estudada.

Por outro lado, é importante destacar que o apoio social pode não trazer necessariamente um efeito positivo para a mudança de comportamento entre os jovens. A informação, o incentivo e os esforços práticos realizados por familiares e amigos podem estar na contramão do interesse e da realidade cultural do adolescente. Toda forma de atividade física deve estar interligada ao sentido que o adolescente emprega nas práticas que se envolve. Neste grupo etário, são notáveis as preocupações sobre a forma do corpo e o controle de peso - razões principais relatadas para a participação de jovens em atividades físicas em estudo de revisão $0^{28}$, principalmente entre as meninas, para as quais a preocupação com o corpo é maior quando comparada aos meninos.

Nos parágrafos anteriores, procuramos ampliar as esferas importantes para o apoio social. Outras escalas e possibilidades metodológicas agregariam conhecimento para a área de apoio social. Outro aspecto importante refere-se a um possível viés de causalidade reversa oriundo do delineamento empregado, em que não podemos determinar se os adolescentes que são fisicamente ativos obtiveram maior apoio social para a prática de atividade física no lazer ou se eles receberam o apoio após o início dessa prática. No entanto, independentemente do momento em que for fornecido o apoio social, o mesmo parece ter grande importância para iniciar ou manter a prática de atividade física.

Futuros trabalhos poderão propor outras formas de estudar o apoio social, observando as diferentes redes sociais dos adolescentes, como a escola e a comunidade, em interação com pais e amigos, a partir das práticas de atividade física em diferentes locais, como bairros, praças, clubes e escola. Contudo, no contexto estudado, o apoio social de familiares e de amigos foi positivamente associado as práticas de atividades físicas no lazer, sugerindo a relevância do incentivo, do convite e da prática conjunta entre os adolescentes.

\section{Conflito de interesses}

Os autores declaram não haver conflitos de interesse.

\section{Contribuição dos autores}

Peixoto MB, Silva ICM e Knuth AG participaram da elaboração do trabalho, processos de análise e redação do artigo. Hallal PC orientou o trabalho desde a sua concepção até as fases de análise e escrita do artigo.

\section{Agradecimentos}

Este artigo faz parte da dissertação de mestrado do primeiro autor deste trabalho e foi realizado junto ao Programa de Pós-Graduação em Epidemiologia da Universidade Federal de Pelotas. Esta "O presente trabalho foi realizado com apoio da Coordenação de Aperfeiçoamento de Pessoal de Nível Superior - Brasil (CAPES).

\section{Referências}

1. Hallal P, Andersen L, Bull F, Guthold R, Haskell W, Ekelund U. Global physical activity levels: surveillance progress, pitfalls, and prospects. Lancet. 2012; 380:247-57.

2. Strong W, Malina R, Blimkie C, Daniels S, Dishman R, Gutin B. Evidence based physical activity for school-age youth. J Pediatr. 2005;146:732-7.

3. Bauman A, Reis R, Sallis J, Wells J, Loos R, Martin B. Correlates of physical activity: why are some people physically active and others not? Lancet. 2012; 380:258-71.

4. Farias Junior J, Lopes A. Comportamento de risco relacionados à saúde em adolescentes. Rev Bras Ciênc Mov. 2004;12(1):7-12.

5. McNeill L, Kreuter M, Subramanian S. Social environment and physical activity: a review of concepts and evidence. Soc Sci Med. 2006;63(4):1011-22.

6. Sallis J, Prochaska J, Taylor W. A review of correlates of physical activity of children and adolescents. Med Sci Sports Exerc. 2000;32(5):963-75.

7. Lisboa T, Silva WR, Alexandre JM, Beltrame TS. Suporte social da família e amigos para a prática de atividade física de adolescentes: uma revisão sistemática. Cad. Saúde Colet. 2018;26(4):351-59.

8. Vrazel J, Saunders R, Wilcox S. An overview and proposed framework of social-environmental influences on the physical-activity behavior of women. Am J Health Promot. 2008;23(1):2-12.

9. Strine T, Chapman D, Balluz L, Mokdad A. Health-related quality of life and health behaviors by social and emotional support. Their relevance to psychiatry and medicine. Soc Psychiatry Psychiatr Epidemiol. 2008;43(2):151-9.

10. Hohepa M, Scragg R, Schofield G, Kolt G, Schaaf D. Social support for youth physical activity: Importance of siblings, parents, friends and school support across a segmented school day. Int J Behav Nutr Phys Act. 2007;4(54).

11. Instituto Brasileiro de Geografia e Estatística. Censo Brasileiro 2010. Rio de Janeiro: IBGE. 2011.

12. WHO - World Health Organization. Young People's Health - a Challenge for Society. Report of a WHO Study Group on Young People and Health for All. Technical Report Series 731. Geneva: WHO, 1986.

13. Silva I, Azevedo M, Gonçalves H. Leisure-Time Physical Activity and Social Support Among Brazilian Adults. J Phys Act Health. 2013;10(6):871-79. 
14. Bastos J,Araújo C, Hallal P. Prevalence of insufficient physical activity and associated factors in Brazilian adolescents. J Phys Act Health. 2008;5(6).777-94.

15. Reis R, Sallis J. Validade e reprodutibilidade da versão brasileira da escala se suporte social para o exercício em adolescentes. Rev Bras Ciênc Mov. 2005;13(2):7-15.

16. Associação Brasileira de Empresas de Pesquisa. Critério Brasil: Critério de Classificação Econômica Brasil 2012: base LSE 2010. [Citado em 2016 abr 2]. Disponível em: <http:// www.abep.org/criterio-brasil>.

17. Wright M, Wilson D, Griffin S, Evans A. A qualitative study of parental modeling and social support for physical activity in underserved adolescents. Health Educ Res 2010;(25)2:224-32.

18. Hallal PC, Knuth AG. Epidemiologia da atividade física e a aproximação necessária com as pesquisas qualitativas. Rev Bras Cienc Esporte. 2011;33(1):181-92.

19. Reis R, Petroski E. Application of the social cognitive theory to predict stages os change in exercise for brazilian adolescents. Rev Bras Cineantropom Desempenho Hum. 2005;7(2):62-8.

20. Reis M, Reis R, Hallal P. Validade e fidedignidade de uma escala de avaliação do apoio social para a atividade física. Rev Saúde Públ. 2011;45(2):294-301.

21. Ricardo LIC, Rombaldi AJ, Otte J, Perez ACA, Azevedo MR. Associação entre apoio social e atividade física no lazer em escolares do ensino médio. Rev Bras Ativ Fís Saúde. 2013;18(2):168-76.

22. Figueira Junior AJ. Influência da família na atividade física de adolescentes. Conexões: Educ. Fís. Esporte e Saúde. 2000;0(4):28-43.
23. Fermino RC; Rech CR, Hino AAF, Añez CRR, Reis RS. Atividade física e fatores associados em adolescentes do ensino médio de Curitiba, Brasil. Rev Saude Publica. 2010;44(6):986-95.

24. Silva I, Azevedo M, Gonçalves H. Leisure-Time Physical Activity and Social Support Among Brazilian Adults. J Phys Act Health. 2013;10(6):871-79.

25. Seabra A, Mendonça D, Thomis M, Anjos L, Maia J. Determinantes biológicos e sócio-culturais associados à prática de atividade física de adolescentes. Cad Saude Publica. 2008;24(4):721-36.

26. Amorim T, Azevedo M, Hallal P. Physical activity levels according to physical and social environmental factors in a sample of adults living in south Brazil. J Phys Act Health. 2010;7 Suppl 2:204-12.

27. Wold B, Hendry L. Social and environmental factors associated with physical activity in young people. In: Biddle S, Sallis JF, Cavill N, editors Young and active? Young people and health-enhancing physical activity - evidence and implications London: Health Education Authority. 1998;119-32.

28. Allender S, Cowburn G, Foster C. Understanding participation in sport and physical activity among children and adults: a review of qualitative studies. Health Educ Res. 2006;21(6):826-35.

Recebido: 02/07/2019

Aprovado: 07/04/2020

\section{Como citar este artigo:}

Peixoto MB, Knuth AG, Silva ICM, Hallal, PC. Apoio social e prática de atividade física no lazer em adolescentes: um estudo de base populacional. Rev Bras Ativ Fís Saúde. 2019;24:e0103. DOI: 10.12820/rbafs.24e0103 